\title{
Eye-associated multiple cranial nerve palsies
}

\begin{abstract}
Multiple cranial nerve paresis (MCNP) can occur due to some syndromes, systemic diseases, extracranial and intracranial pathologies. The paresies including cranial nerves III, IV, V, VI, and VII are eye-associated MCNP. The common causes of eyeassociated MCNP often include cavernous sinus syndrome, superior orbital fissure syndrome, orbital apex syndrome, and cerebellopontine angle syndrome. Clinical approach to MCNP includes the careful examination for generalized limitation in various gaze positions, proptosis, decreased corneal and facial sensation, conjunctival injection, ptosis, anisocoria, and cerebellar signs. In this review, we aim to briefly remind the main causes of MCNP associated with the eye.
\end{abstract}

Keywords: multiple, combined, cranial nerve, palsy, paresis, eye-associated
Volume 9 Issue 3 - 2019

\author{
Burak Turgut,' Onur Çatak, ${ }^{2}$ Sabiha Güngör \\ Kobat, ${ }^{3}$ \\ 'Onsekiz Mart University, Faculty of Medicine, Department of \\ Ophthalmology, Turkey \\ ${ }^{2}$ Firat University, Faculty of Medicine, Department of \\ Ophthalmology, Turkey \\ ${ }^{3}$ Elazig Training and Research Hospital of Health Sciences \\ University, Turkey
}

Correspondence: Burak Turgut, Onsekiz Mart University, Faculty of Medicine, Department of Ophthalmology, Canakkale, Turkey,Tel+90533 7I28389, Email burakturgut@comu.edu.tr

Received: April 04, 2019 | Published: May 22, 2019

Abbreviations: $\mathrm{CN}$, cranial nerve; MCNP, multiple cranial nerve paresis; CSS, cavernous sinus syndrome; SOF, superior orbital fissure; SOFS, superior orbital fissure syndrome; OA, orbital apex; OAS, orbital apex syndrome; CPA, cerebellopontine angle; CPAS, cerebellopontine angle syndrome; THS, Tolosa-Hunt syndrome; CS, cavernous sinus; CCF, carotid-cavernous fistula; BoS, base of skull; LMS, lateral medullary syndrome; PICA, posterior inferior cerebellar artery

\section{Introduction}

Cranial nerve $(\mathrm{CN})$ paresies are neuropathies which might be classified as isolated/single or combined/multiple, unilateral or bilateral and painless or painful paresis. Additionally, they may be presented acute, subacute or recurrent manner. Combined or multiple cranial nerve paresis (MCNP) can occur due to a variety of different causes such as some syndromes or systemic diseases, extracranial or intracranial pathologies (brain stem, meninx, and base of the skull (BoS). ${ }^{1-6} \mathrm{CN}$ paresies including CN III (n.oculomotorius), IV (n.trochlearis), V (n.trigeminus), VI (n.abducens) and VII (n.facialis) are eye-associated MCNP. ${ }^{6-9}$ The common causes of eye-associated MCNP often include cavernous sinus syndrome (CSS), superior orbital fissure syndrome (SOFS), orbital apex syndrome (OAS) and cerebellopontine angle syndrome (CPAS).$^{8-18}$ A recent report including the largest series of 979 cases of MCNP by Kaene demonstrates that the most common causes of MNCP are tumors with a percent of $30 .{ }^{4}$ The other common causes by frequency order are infarctions, trauma, infection, Guillain-Barré syndrome, Fisher syndrome, idiopathic cavernous sinusitis, surgery, multiple sclerosis, demyelinating encephalomyelitis, and diabetes mellitus. In this publication, it has been reported that the most common tumor was schwannoma with a percent of 17. The other tumoral causes of MNCP are metastases, meningioma, lymphoma, pontine glioma, nasopharyngeal carcinoma, pituitary adenoma, chordoma, leukemia, epidermoid tumor, and glomus jugulare tumor, respectively. Kaene reported that the common pathologic sites were cavernous sinus (CS) $(25 \%)$, clivus and BoS (13\%), subarachnoid space (10\%) and cerebellopontine angle (CPA) $(8 \%)$. CN which is most commonly involved is CN VI followed by $\mathrm{CN} \mathrm{V}, \mathrm{IV}^{4}$

\section{Cavernous sinus syndrome (CSS)}

The cavernous sinus (CS) is a venous plexus located between the periosteal and dural layers of the meninx and at the central BoS, on both sides of the sella turcica (pituitary). Structures passing through $\mathrm{CS}$ are the internal carotid artery, its sympathetic plexus and CN, III, IV, VI, V1 (n.ophthalmicus) and V2 (n.maxillaris) and the superior and inferior orbital veins (SOV and IOV). ${ }^{1-9,15-18}$ Cavernous sinus syndrome is characterized by MCNP manifesting with ophthalmoplegia, ptosis, and facial sensory loss, proptosis, orbital (ocular and conjunctival) congestion, sympathetic disturbance and Horner's syndrome due to MCNP of the CN III, IV, and VI responsible for ocular movements and pupillary function, and at least one branch of the CN V. However, CSS does not involve the optic nerve. The most common causes of CSS are neoplastic (metastatic including head and neck tumors and primary tumors such as lymphoma), traumatic, vascular (aneurysms, fistulas, and thrombosis), congenital, infectious (fungal infection), inflammatory or granulomatous pathologies involving CS, and Tolosa Hunt syndrome (THS), the idiopathic granulomatous inflammation involving CS..$^{1-9,15-18}$ In pure CSS, the involvement of CNs III, IV, VI and V1, V2, V3 (n.mandibularis) and Horner's syndrome is present. The anterior CSS only involves CN V1 branch. The middle CSS is caused by the involvement of CN V1 and V2. The posterior CSS occurs in whole CN V involvement. ${ }^{14}$ In CSS associated with Horner's syndrome, the pain is present in contrast to SOFS. ${ }^{15}$ The frequency of the involvement of four CNs has been reported as is in about $78 \%$ of cases. ${ }^{19}$

\section{Superior orbital fissure syndrome (SOFS)}

Superior orbital fissure (SOF) lies at the back of the orbit between the lesser and greater wing of the sphenoid bone. It contains the SOV and IOV, superior and inferior branches of CN III, IV, VI, V1 and its branches including lacrimal, frontal, supraorbital, supratrochlear and nasociliary nerves. SOFS which is also known as Rochen-Duvigneaud syndrome is characterized by retro-orbital paralysis of EOMs and impairment of the CNV1 without optic neuropathy. SOFS is a symptom complex caused by compression of structures which exist in SOF just anterior to the orbital apex..$^{1-4,8-14}$ The main difference of SOFS from OAS is no optic nerve involvement in SOFS. ${ }^{11}$ In SOFS, CNs III, 
IV, VI and V1 is involved. It is known that the most common cause of SOFS is trauma (craniomaxillofacial injury) including motorcycle accidents, zygomatic and orbital fractures. Other causes of SOFS are tumors including lymphoma and rhabdomyosarcoma, infectious diseases including syphilis (syphilitic periostitis), meningitis, sinusitis, herpes zoster, ischemic, vasculitic or inflammatory diseases including THS, sarcoidosis, systemic lupus eritematosus (SLE), Wegener's granulomatosis, polyarteritis nodosa, or temporal arteritis and vascular events including carotid-cavernous fistulas (CCF), retroorbital haematoma and carotid aneurysm. However, it may also be idiopathic. ${ }^{1-4,8-14}$ In SOFS, the compression and inflammation of neural structures which is responsible for main clinical symptoms are caused by a bony fragment from facial fractures or any edema, bleeding or mass in SOF. CN VI is most commonly damaged because of its location within the central SOF and close to the greater wing. $\mathrm{CN}$ IV is the least commonly involved $\mathrm{CN}$ because of its protection by the common tendinous ring. ${ }^{1-4,8-14}$ The main clinical features of SOFS include ophthalmoplegia due to damage to CN III, IV and VI, ptosis due to CN III injury and loss of sympathetic input, proptosis due to decreased tension in the EOMs with loss of innervation, fixed dilated pupil due to loss of parasympathetic innervation of the pupil by the CN III, lacrimal hyposecretion and eyelid or forehead anaesthesia and decreased corneal sensation due to damage to $\mathrm{CN} \mathrm{V1}$, chemosis and bruits caused by vascular congestion and occasionally visual loss due to mechanical compression of CN II (n.opticus). The proptosis, eyelid swelling, and chemosis indicate significant orbital masses. Additionally, the patients with SOFS caused by a facial trauma may show the findings such as subconjunctival hemorrhage, periorbital ecchymosis, and soft tissue contusion attributed to trauma. The partial SOFS is the involvement of the central sector associated with isolated CN III, CN VI, and nasociliary nerve..$^{1-4,8-14}$

\section{Tolosa-hunt syndrome (THS)}

Tolosa-Hunt syndrome is a cause of the painful ophthalmoplegia in the fifth decade with unknown etiology located in SOF and anterior CSs. The cause of pain is granulomatous inflammation due to the infiltration of the septas or walls of SOF or CS by lymphocytes and macrophages. The good response of the pain to the steroid treatment within 72 hours is critical for diagnosis of THS. Common diagnostic criteria for THS are severe unilateral peri- or retro-orbital pain in a perforating manner, ipsilateral ophthalmoplegia with or without periarterial sympathetic fiber and optic nerve involvement and any local or systemic pathology, exclusion of traumatic, infective, vascular, neoplastic, metabolic, and inflammatory causes, and episodes with spontaneous remissions. The paralysis of one or more of the CNs III, IV, and CN VI occur. However, CN V1 and V2 may be affected. The most affected $\mathrm{CN}$ is $\mathrm{CN}$ III. If inflammation reaches to OA, CN II dysfunction may be developed. Horner syndrome may sometimes be associated with THS due to the involvement of carotid sympathetic fibers. Pupillary dysfunction may occur in some cases because of the involvement of parasympathetic fibers of CN III. ${ }^{20-21}$

\section{Orbital apex syndrome (OAS)}

The orbital apex $(\mathrm{OA})$ is the most posterior part of the pyramidalshaped orbit at the craniofacial junction. OA includes the region (tendinous ring) where the rectus EOMs origins and also the entry of neuro-vascular structures transmitted from the intracranial compartment into the orbit through bony apertures (optic canal, SOF and inferior orbital fissure). ${ }^{1-4,8-11,17,22}$ Orbital apex syndrome is also known as Jacod syndrome. OAS is characterized by the involvement of CNs II, III, IV and VI. The clinical characteristics of OAS are vision loss (if CN II involvement is present), optic neuropathy and ophthalmoplegia. The etiologies of OAS are neoplastic pathologies (nasopharyngeal carcinoma, hematological tumors, neural tumors, lymphoma, metastasis or middle cranial fossa (near the apex of the orbit), inflammatory causes such as idiopathic orbital inflammation, collagen vascular disease, sarcoidosis, systemic lupus erythematosus, granulomatosis with polyangiitis, giant cell arteritis, thyroid disease, iatrogenic causes (following sinonasal surgery), orbital apex fracture, vascular events like carotid aneurysm, trauma, mucocele, fungal infections such as Aspergillus and Mucormycosis. OAS is separated from SOFS with occurring a lesion immediately anterior to the orbital apex. ${ }^{1-4,8-11,17,22}$

\section{Cerebellopontine angle syndrome (CPAS)}

The cerebellopontine angle (CPA) is a triangular area situated at inferior to the tentorium, lateral to the pons, and ventral to the cerebellum. CPAS is characterized by MNCP including CN V, VII, VIII and cerebellar signs. Its etiology includes vascular (intracavernous sinus carotid aneurysm or post-cerebral aneurysm, cavernous sinus thrombosis, migraine, giant cell arteritis), inflammatory (THS, meningitis, syphilis, tuberculosis and herpes zoster infection, Wegener's granulomatosis, Sarcoidosis), tumoral (primarypituitary adenoma, meningioma, craniopharyngioma, secondary to nasopharyngeal carcinoma, lymphoma and distant metastasis), traumatic pathologies and CCF. CPAS arises from the closeness of the CPA to specific CNs. ${ }^{1-4,8-10,12,13}$ The presentation includes unilateral hearing loss (most common), speech impediments, imbalance, vertigo, tinnitus, disequilibrium, tremors or slowly progressive deafness, other loss of motor control. CPAS is usually due to an acoustic schwannoma arising from the vestibular nerve. Other tumoral causes of the CPAS are pontine glioma, meningioma, epidermoid and cerebellar astrocytoma. Diplopia due to CN VI palsy, papilledema due to raised intracranial pressure, fine rapid gaze-evoked nystagmus to the side opposite the lesion, and ipsilateral low frequency and large amplitude vertical nystagmus, ipsilateral corneal reflex loss and facial weakness may associated to above mentioned signs. ${ }^{1-4,8-10,12,13}$

\section{Lateral medullary syndrome (LMS)}

Lateral medullary syndrome (LMS) is a neurological symptom complex due to ischemia from atherothrombotic occlusion most commonly in the vertebral artery or the posterior inferior cerebellar artery (PICA) in the brainstem. LMS etiology includes stroke and multiple sclerosis. ${ }^{8,23}$ LMS is also called Wallenberg's syndrome, PICA syndrome, and vertebral artery syndrome. In LMS, CNs V, VIII, IX and X were involved. It is characterized by contralaterally sensory deficits affecting the trunk and extremities, and ipsilateral sensory deficits affecting the face and CNs. ${ }^{8,23}$ Clinical features include contralaterally impaired pain or facial sensation and temperature sensation in the arms and legs (due to the involvement of $\mathrm{CN} \mathrm{V}$ and spinothalamic tract, respectively), ipsilateral impaired taste sensation due to the involvement of the tractus and nucleus solitarius, nystagmus caused by the involvement of CN VIII, dysarthria, and dysphonia, nausea/vomiting, dizziness, nystagmus commonly associated with vertigo spells, falling caused by the involvement of Deiters' nucleus, imbalance in walking or maintaining (ataxia) caused by the damage to the cerebellum or the inferior cerebellar peduncle, dysphagia or difficulty swallowing caused by the involvement of the 
nucleus ambiguus that supplies CNs IX and X (n.glossopharyngeus and n.vagus), and Horner's syndrome due to damage of the hypothalamospinal fibers and sympathetic tract. ${ }^{8,23}$

\section{Conclusion}

Clinical approach to MCNP includes the careful examination for limitations in different gaze positions, proptosis, decreased corneal and facial sensation, conjunctival injection, ptosis, anisocoria, and cerebellar signs.

\section{Acknowledgments}

None.

\section{Conflicts of interest}

The authors declare that there is no conflict of interest regarding the publication of this paper.

\section{Authorship contributions}

All authors have equally contributed to the concept and design, data collection, literature Search in the work and writing of the manuscript.

\section{References}

1. Campbell WW. Brainstem and multiple cranial nerve syndromes. Dejong's The Neurologic Examination. (sixth edition), Philadelphia: Lippincott Williams and Wilkins; 2005. p. 277-296.

2. Martin TJ, MD, Corbett JJ. Ocular Motility Disorders: Cranial Nerve Palsies. Practical Neuroophthalmology, McGraw-Hill Education LLC, 2013. China.

3. Carroll CG, Campbell WW. Multiple cranial neuropathies. Semin Neurol. 2009;29(1):53-65.

4. Keane JR. Multiple cranial nerve palsies. Arch Neurol. 2005;62(11):1714 1717.

5. Kumar K, Ahmed R, Bajantri B, et al. Tumors Presenting as Multiple Cranial Nerve Palsies. Case Rep Neurol. 2017;9(1):54-61.

6. Ross M, Bursztyn L, Superstein R, et al. Multiple Cranial Nerve Palsies in Giant Cell Arteritis. J Neuroophthalmol. 2017;37(4):398-400.

7. Shin SY, Lee JM. A case of multiple cranial nerve palsies as the initial ophthalmic presentation of antiphospholipid syndrome. Korean $J$ Ophthalmol. 2006;20(1):76-78.

8. Wong TY, Sng C, Lim L. Neuroophthalmology. The Ophthalmology Examinations Review (Second edition), Singapore: World Scientific Publishing Co. Pte. Ltd, 2011. p. 267-343.
9. Sharpe JA, Wong AM, Fouladvand M. Ocular motor nerve palsies: implications for diagnosis and mechanisms of repair. Prog Brain Res. 2008;171:59-66.

10. Walsh F, Hoyt W. Superior Orbital Fissure Syndrome. Walsh and Hoyt's clinical neuro-ophthalmology (Volume 1, 2nd edition), USA: Lippincott Williams \& Wilkins; 1969. p. 398-399.

11. Yeh S, Foroozan R. Orbital apex syndrome. Curr Opin Ophthalmol. 2004;15(6):490-498

12. Samii M, Gerganov VM. Tumors of the cerebellopontine angle. In: Grisold W, Soffietti R, editors, Handbook of Clinical Neurology, 2012. p. 633-639.

13. Furman JM, Mccall AA. Otoneurologic Manifestations of Otologic and Systemic Disease. In: Aminoff MJ, Josephson SA, editors, Aminoff's Neurology and General Medicine (Fifth Edition), Academic Press; 2014. p. $461-478$.

14. Bowerman JE. The superior orbital fissure syndrome complicating fractures of the facial skeleton. Br J Oral Surg. 1969;7(1):1-6.

15. Lee JH, Lee HK, Park JK, et al. Cavernous sinus syndrome: clinical features and differential diagnosis with MR imaging. AJR Am J Roentgenol. 2003;181(2):583-590.

16. Lazaridou M, Bourlidou E, Kontos K, et al. Carotid-Cavernous Fistula as a Complication of Facial Trauma: A Case Report. Craniomaxillofacial Trauma \& Reconstruction. 2015;8(3):239-245.

17. Bone I, Hadley D. Syndromes of the orbital fissure, cavernous sinus, cerebello-pontine angle, and skull base. J Neurol Neurosurg Psychiatry. 2005;76(Suppl 3):iii29-iii38.

18. Bhatkar S, Goyal MK, Takkar A, et al. Cavernous sinus syndrome: A prospective study of 73 cases at a tertiary care centre in Northern India. Clin Neurol Neurosurg. 2017;155:63-69.

19. Lin CC, Tsai JJ. Relationship between the number of involved cranial nerves and the percentage of lesions located in the cavernous sinus. Eur Neurol. 2003;49(2):98-102.

20. Amrutkar C, Burton EV. Tolosa-Hunt Syndrome. StatPearls. Treasure Island (FL): StatPearls Publishing; 2019.

21. Waldman SD. Pain of Ocular and Periocular Origin. In: Waldman SD, Bloch JI, WB Saunders, editors, Pain Management, 2007. p. 523-537.

22. Aryasit O, Preechawai P, Aui-Aree N. Clinical presentation, aetiology and prognosis of orbital apex syndrome. Orbit. 2013;32(2):91-94.

23. Ogawa K, Suzuki Y, Oishi M, et al. Clinical study of 46 patients with lateral medullary infarction. J Stroke Cerebrovasc Dis. 2015;24(5):1065-1074. 